\title{
Revision of the south Asian amisegine genus Cladobethylus Kieffer, 1922 (Hymenoptera, Chrysididae, Amiseginae)
}

\author{
Lynn S. Kimsey ${ }^{1}$
}

I Bohart Museum of Entomology, Department of Entomology and Nematology, University of California, Davis, California, 95616, USA

Corresponding author: Lynn S. Kimsey (lskimsey@ucdavis.edu)

Academic editor: Michael Ohl | Received 11 March 2019 | Accepted 15 June 2019 | Published 28 June 2019

http://zoobank.org/B8F3FB3E-7237-49E7-9AA7-1AAC9EB4C787

Citation: Kimsey LS (2019) Revision of the south Asian amisegine genus Cladobethylus Kieffer, 1922 (Hymenoptera, Chrysididae, Amiseginae). Journal of Hymenoptera Research 70: 41-64. https://doi.org/10.3897/jhr.70.34206

\begin{abstract}
The south Asian genus Cladobethylus Kieffer, 1922, is reviewed, with a key to the species. Five new species are described, one from the island of Borneo ( $\mathrm{Cl}$. darlingi), two from Thailand $(\mathrm{Cl}$. densepunctatus and $\mathrm{Cl}$. thailandicus), one from Korea (Cl. koreensis), and one from Sumatra (acehensis).
\end{abstract}

\section{Keywords}

Vietnam, Eurycantha insularis, Phasmatodea, Phasmatidae

\section{Introduction}

Cladobethylus Kieffer, 1922, is one of the most widespread and speciose of the south Asian amisegine genera, with 11 species, including the ones newly described below. There are undoubtedly more species to be discovered and it would not be surprising to find species in southern China, Cambodia and other islands in Indonesia, such as Sulawesi. The current distribution of the genus is probably more a reflection of where collecting has been done and the techniques used than the actual distribution. Based on personal observation, as with most other amisegines, males are more commonly collected, particularly in Malaise traps and by sweep netting. Females are rarer in collections, and are more likely to be collected in bowl and pitfall traps. This may have 
more to do with the environments the two sexes typically inhabit than any actual differences in numbers. Females may spend most of their time searching in and on leaf litter for walking stick eggs, whereas males fly and sit on vegetation above the ground searching for females, and are more likely to be caught in a Malaise trap.

The majority of Cladobethylus species are moderate-sized amisegine wasps ranging from 2-4 mm long. However, one species, Cl. insularis Kimsey \& Dewhurst, from Papua New Guinea is much larger, ranging from $5-7 \mathrm{~mm}$ in length. This is also the only species, where a host is known. It parasitizes the eggs of Eurycantha insularis Lucas (Phasmatodea: Phasmatidae) (Kimsey et al. 2012).

\section{Materials and methods}

Specimens were borrowed from and/or types are deposited in the following institutions, which are given in the descriptions below using their acronyms: AEI - American Entomological Institute, Logan, Utah, USA; BME - Bohart Museum of Entomology, University of California, Davis, USA; BMNH - The Natural History Museum, London, U.K.; BPBM - B.P. Bishop Museum, Honolulu, Hawaii, USA; MZB - Museum Zoologi Bogor, Chibinong, Indonesia; QSBG - Queen Sirikit Botanical Garden, Chiang Mai, Thailand; ROM - Royal Ontario Museum, Toronto, Ontario, Canada; USNM - U. S. National Museum of Natural History, Washington, D. C., USA.

Morphological terminology follows that used by Kimsey and Bohart (1991). The acronym MOD is used for midocellus diameter, which is the width of the midocellus from side to side in front view. The interocular distance is measured across the narrowest part of the face in front view, using the greatest width of the eye in front view for comparison. Antennal articles are measured at the point of greatest breadth and compared with the total length of the article. Postocular distance is measured across the greatest width of the head laterally behind the eye margin in dorsal view.

\section{Systematics}

\section{Genus Cladobethylus Kieffer, 1922}

Cladobethylus Kieffer, 1922: 67. Type species: Cladobethylus cruciger Kieffer, 1922:69. Original designation.

Diagnosis. The most distinctive feature of the genus Cladobethylus is the impunctate welt that extends along the midline of the vertex from near the midocellus to the occipital carina. This feature is only shared with species of Magdalium Kimsey and Senesega Kimsey, although it is less well developed in these genera. Cladobethylus can be distinguished from those genera by the lack of an omaulus on the mesopleuron and the usually biangulate genal area, which is evenly curved in Magdalium and Senesega. 
In addition, Cladobethylus species have at most a weakly defined scrobal sulcus. The scrobal sulcus in Magdalium and Senesega is well defined.

Description. Vertex with low impunctate welt along midline from midocellus to occiput; eye with short sparse setulae; scapal basin usually with at least some crossridging; malar space with vertical sulcus extending from ventral eye margin to mandibular articulation; occipital carina present; posterior margin of head behind eye usually biangulate in side view; male flagellum elongate and cylindrical; female flagellum short, fusiform and usually flattened on one surface; pronotum with narrow posteromedial groove; mesopleuron without scrobal sulcus or omaulus; scutum with notauli and parapsides; both sexes fully winged, forewing Rs extended by evenly curved dark streak, costal cell widest medially, stigma large and broad, $\mathrm{R}_{1}$ not indicated, medial vein arising before cu-a; propodeum with long dorsal surface and abrupt posterior declivity, lateral propodeal angle undeveloped; hindcoxa with dorsobasal carina; tarsal claws edentate in females, with one small subbasal tooth and subbasal angle in males.

Distribution (Fig. 1). Tropical Asia: Sri Lanka, New Guinea, Malaysia, Vietnam, Sarawak, Philippines, Indonesia (Sumatra), Borneo, South Korea and Japan.

Hosts. Cladobethylus insularis was reared from eggs of the oil palm stick insect, Eurycantha insularis Lucas (Phasmatodea: Phasmatidae), in Papua New Guinea (Kimsey et al. 2012).

\section{Key to the Species of Cladobethylus*}

1 Four visible metasomal segments; flagellum tapering, broadest submedially; females

- $\quad$ Five visible metasomal segments; flagellum slender, parallel-sided; males.....11

\section{Females}

2 Metasomal tergum II with punctures $0-2$ puncture diameters apart ..........3

- Metasomal tergum II with tiny punctures 5-10 or more puncture tiny diameters apart ................................................................................6

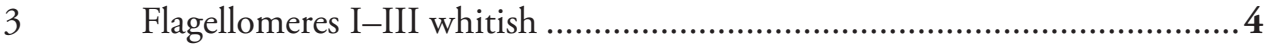

- $\quad$ Flagellomeres I-III dark brown ..........................................................5

4 Malar space 3.5 MOD wide; femoral apices whitish, contrasting with rest of femur; body with bluish metallic highlights, particularly dorsally; Papua New Guinea Cl. insularis Kimsey

- Malar space 4.5 MOD wide; femoral apices not whitish or contrasting with rest of femur; body without bluish metallic highlights; Papua New Guinea ...

Cl. aquilus Kimsey

* Females are unknown for $\mathrm{Cl}$. acehensis and $\mathrm{Cl}$. ceylonicus, and males are unknown for $\mathrm{Cl}$. japonicus and Cl. koreensis. 
Underside of head with occipital fovea on either side of occipital suture; body with bluish metallic highlights, particularly dorsally; Philippines

.. Cl. cruciger Kieffer

- $\quad$ Underside of head with occipital fovea on either side of occipital suture; body with bluish metallic highlights, particularly dorsally; Thailand, Viet Nam .... Cl. densepunctatus Kimsey, sp. nov. Underside of head without occipital fovea on either side of occipital suture; metapleuron and propodeal side cross-ridged or wrinkled. Underside of head with occipital fovea on either side of occipital suture; metapleuron and propodeal side smooth without cross-ridges or wrinkles ...........8 Malar space less than 4 MOD wide; ocular setulae less than 1 MOD long; Thailand Cl. thailandicus Kimsey, sp. nov.

- Malar space more than 4.5 MOD wide; ocular setulae 1 MOD long or longer; body with bluish metallic highlights, particularly dorsally; Korea

Cl. koreensis Kimsey, sp. nov.

Flagellomere I less than $1.5 \times$ as long as broad; flagellomere II broader than long

Flagellomere I twice as long as broad or longer; flagellomere II as long as broad or longer.....

Malar space more than 4 MOD wide; flagellomere X twice as long as broad; Malaysia.

Cl. gilbus Kimsey

Malar space less than 4 MOD wide; flagellomere X 1.5-1.6x as long as broad; Borneo. Cl. sarawakensis Kimsey, sp. nov. Legs and antennae yellow; malar space less than 4.5 MOD wide; body without metallic highlights; Borneo

Cl. darlingi Kimsey, sp. nov. Legs and antennae brown; malar space more than 4.5 MOD wide; body with metallic highlights; Japan

Cl. japonicus Kimsey

Males

11 Metasomal tergum II with punctures 0-2 puncture diameters apart 12 Metasomal tergum II with tiny punctures 10 or more puncture diameters apart. 15

12 Malar space less than 3 MOD wide; post ocular distance 2 MOD wide; body length 5-7 mm; Papua New Guinea.......Cl. insularis Kimsey \& Dewhurst Malar space 3 MOD wide or wider; post ocular distance less than $2 \mathrm{MOD}$ wide; body length less than $4.5 \mathrm{~mm}$

13 Face and malar space coarsely punctate; head dorsolaterally strongly angulate behind eye in lateral view; Thailand, Viet Nam

Cl. densepunctatus Kimsey, sp. nov.

- $\quad$ Face and malar space largely impunctate, with few scattered punctures; head dorsolaterally rounded or obtusely angulate behind eye in lateral view.......14 
14 Malar space more than 3.5 MOD wide; postocular distance 1.5 MOD wide or wider; Sri Lanka. Cl. ceylonicus Krombein

- Malar space 3 MOD wide; postocular distance less than 1.5 MOD wide; Papua New Guinea Cl. aquilus Kimsey

15 Postocular distance more than 2 MOD wide; scapal basin without cross ridging; flagellomere $\mathrm{X}$ less than $5.5 \times$ as long as broad; Thailand

Cl. thailandicus Kimsey, sp. nov.

- $\quad$ Postocular distance less than 1.6 MOD wide; scapal basin with zones of crossridging; flagellomere $\mathrm{X}$ more than $5.5 \times$ as long as broad

16 Malar space less than 3 MOD wide; postocular distance less than 1 MOD wide; Malaysia.

- Malar space 3 or more MOD wide; postocular distance 1 MOD wide or wider

17 Flagellomere I $4 \times$ as long as broad or longer; Sumatra

Cl. acebensis Kimsey, sp. nov.

- $\quad$ Flagellomere I 3.5x as long as broad or shorter 18

18 Least interocular distance twice greatest eye width or more in front view; Borneo Cl. darlingi Kimsey, sp. nov.

- $\quad$ Least interocular distance $1.5 \times$ greatest eye width or less, in front view; Philippines

Cl. cruciger Kieffer

\section{Cladobethylus acehensis Kimsey, sp. nov.}

http://zoobank.org/9F6DC8C1-143A-4F1B-9B6E-9FCFA5C9A98B

Figs 2, 3

Diagnosis. This species is part of the group that has a nearly impunctate second metasomal tergum, including $\mathrm{Cl}$. darlingi, $\mathrm{Cl}$. densepunctatus, $\mathrm{Cl}$. gilbus and $\mathrm{Cl}$. philippinensis. It can be distinguished from these and other species by the combination of the pale antenna, flagellomere I $4 \times$ as long as broad or longer, flagellomere II $1.5 \times$ as long as broad or shorter, flagellomere XI $6.5 \times$ as long as broad, and flagellomeres VII-X asymmetrical. Other diagnostic features include the long malar space, slightly less than 4 midocellar diameters wide, and body with bluish highlights.

Male description. Body (Figs 2, 3) length 3.0-4.0 mm. Head. frons with punctures 2-4 puncture diameters apart; scapal basin polished and impunctate, with short, sublateral group of transverse ridges; clypeal apicomedial margin slightly concave; subantennal distance 1 MOD long; malar space 3.8 MOD long; head slightly broader than long, interocular distance twice eye width in front view; postocular distance 1.5 midocellar diameters wide; vertex rounded posterolaterally in dorsal view; midocellus 2 MOD from ocular margin; ocelli arranged in equilateral triangle; hindocelli separated from ocular margin by 1.0-1.1 diameters; apical flagellomeres asymmetrical, broadest medially, appearing slightly lobed on one side; flagellomere I $4.2 \times$ as long as 

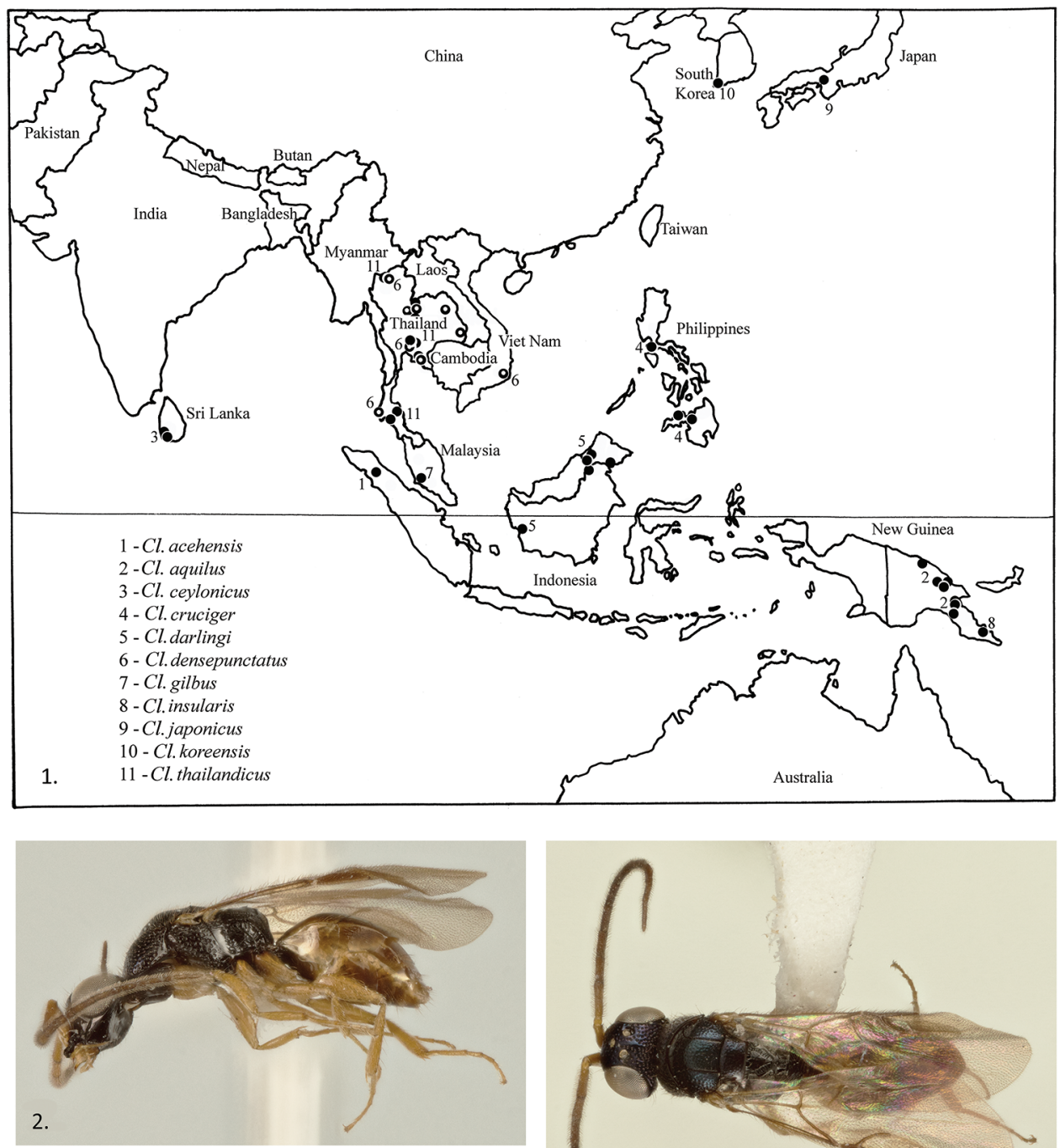

Cl. acehensis

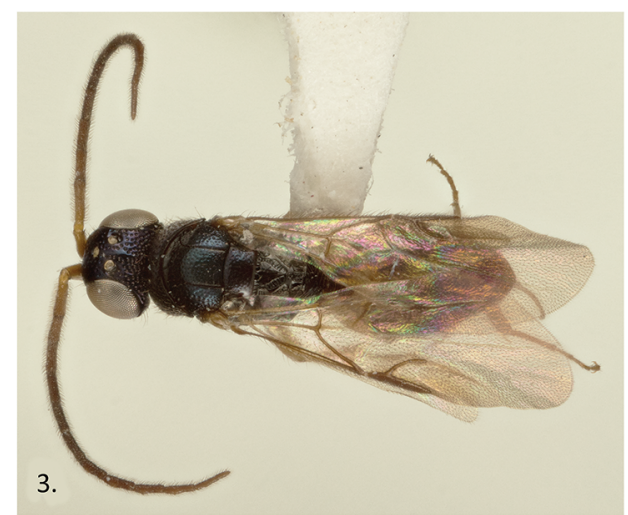

Figures I-3. I Distribution map of Cladobethylus species 2, 3 Cladobethylus acehensis male 2 Lateral view 3 dorsal view.

broad, setae $0.5 \times$ as long as flagellomere breadth; flagellomere II $2.4 \times$ as long as broad; flagellomere IX $6.5 \times$ as long as broad; flagellomeres VIII-X slightly lobate submedially. Mesosoma. Pronotum $0.9 \times$ as long as scutum in dorsal view; mesopleural punctures 1 puncture diameter apart; metapleuron polished, impunctate; propodeal side polished impunctate, posteriorly with subrectangular medial enclosure. Metasoma. Tergum I nearly impunctate; terga II-IV with tiny punctures, 5-10 puncture diameters apart or 
more, with impunctate apical margin. Color. Body black, with metallic blue highlights on dorsum of head, pronotum and scutum; legs and antennae yellow; wing membrane brown tinted, with dark brown veins.

Female. Unknown.

Type material. Holotype male: Indonesia: Sumatra, Aceh, Gunung Lauser Natl. Pk., Ketambe Res. Sta. xi/1989, IIS890004, D.C. Darling (MBZ). Paratypes: 24 males same locality data as holotype; 8 males: 5 Sept. 1989, screen sweep, B. Hubley and D. C. Darling, ROM893044; 1 male: 22-30 Sept. 1989, D. C. Darling, Malaise trap with pans, IIS890003; 1 male: 1-30 Oct. 1989, Malaise trap with pans, D.C. Darling, IIS890007; 3 males: Nov. 1989, Malaise trap, D. C. Darling, IIS890006; 1 male: Nov. 1989, D. C. Darling, IIS890005; 1 male: 1-30 Nov. 1989, D.C. Darling, Malaise trap with pans, IIS890012; 1 male: 1-31 Dec. 1989, Malaise trap with pans, D.C. Darling, IIS890013; 1 male: 1-31 Jan. 1990, Malaise trap, D.C. Darling, IIS900001; 2 males: 1-31 Jan. 1990, Malaise trap, D.C. Darling, IIS900005; 2 males: 1-28 Feb. 1990, Malaise trap, D.C. Darling, IIS900009; 1 male: 1-28 Feb. 1990, Malaise trap, D.C. Darling, IIS900011; 2 males: 1-31 Mar. 1990, Malaise trap, D.C. Darling, IIS900021 (BME, MBZ, ROM).

Distribution. Indonesia: Sumatra, Aceh, Gunung Lauser National Park.

Etymology. The species is named after Aceh Province on the island of Sumatra, Indonesia, where it was collected.

\section{Cladobethylus aquilus Kimsey}

Figs 4-7

Cladobethylus aquilus Kimsey 1986: 157. Holotype male; New Guinea: Bulolo (AEI).

Diagnosis. Cladobethylus aquilus males most closely resemble those of $\mathrm{Cl}$. ceylonicus and $\mathrm{Cl}$. insularis based on flagellomere I $4.0-4.5 \times$ as long as broad, the dark brown antenna, nearly impunctate metasomal tergum II, and reduced cross ridging in the scapal basin. However, Cl. aquilus can be distinguished from those and other species by the combination of the longer subantennal distance, lack of metallic blue highlights, slightly medially lobate apical flagellomeres, and flagellomere XI $6 \times$ as long as broad. Females most closely resemble those of $\mathrm{Cl}$. insularis and $\mathrm{Cl}$. thailandicus based on the bicolored antenna and flagellomere II about as long as broad. They can be distinguished from these and other species by the scapal basin lacking cross ridges, bicolored legs and densely punctate metasomal tergum II.

Male description. Body (Figs 4, 5). Length 3.0-4.0 mm. Head. Frons with punctures $0.5-1.0$ puncture diameter apart; scapal basin impunctate, with narrow band of cross-ridges sublaterally; clypeus long and subtruncate apicomedially; subantennal distance $1 \mathrm{MOD}$ long; malar space 3.4 MOD long; gular area flattened, without depression or pits; head $0.9 \times$ as long as broad, interocular distance twice eye width in front view; postocular distance 1.4 midocellar diameters wide; vertex 
broadly rounded posterolaterally in dorsal view; midocellus 2.2 MOD from ocular margin; ocelli arranged in isosceles triangle; hindocelli separated from ocular margin by 1.0 diameters; flagellomere I $4 \times$ as long as broad, setae $0.5 \times$ as long as flagellomere breadth; flagellomere II $2.8 \times$ as long as broad; flagellomere IX $6 \times$ as long as broad; flagellomeres VIII-X slightly lobate submedially. Mesosoma. Pronotum medially $0.8 \times$ as long as scutum in dorsal view; mesopleural punctures contiguous to 0.5 puncture diameter apart; metapleuron polished, impunctate; propodeum laterally polished, impunctate, posteriorly with posteromedial subrectangular enclosure. Metasoma. Tergum I punctures 5-10 puncture diameters apart; tergum II with punctures, 1-2 puncture diameters apart, separated medially by impunctate medial stripe; tergum III-IV punctures 1-2 puncture diameters apart. Color. Body black, without metallic highlights; legs yellow; antenna brown to blackish; wing membrane light browntinted, with dark brown veins.

Female description. Body (Figs 6, 7). Length. $4.0 \mathrm{~mm}$. Head. Frons punctures contiguous to 0.5 puncture diameter apart; scapal basin impunctate, with narrow vertical band of cross-ridges submedially; clypeus short in front view, projecting ventrally, projection truncate apicomedially; subantennal distance $0.8 \mathrm{MOD}$ wide; malar space 4.5 MOD long; head $0.9 \times$ as long as broad, interocular distance $1.2 \times$ eye width in front view; postocular distance 1 midocellar diameter; vertex rounded angulate posterolaterally in dorsal view; midocellus $2.3 \mathrm{MOD}$ from ocular margin; ocelli arranged in nearly equilateral triangle; hindocelli separated from ocular margin by 1 diameter; flagellomeres appearing somewhat bead-like, broader medially than apically; flagellomere I twice as long as broad; flagellomere II as long as broad; flagellomere X twice as long as broad. Mesosoma. Mesopleural punctation contiguous; metapleuron polished with metapleural-propodeal suture foveate; propodeum polished below wing. Metasoma. Tergum I punctures $2-5$ puncture diameters apart with impunctate medial stripe; tergum II with punctures, 1-2 puncture diameters apart, separated medially by impunctate medial stripe; tergum III-IV punctures 1-2 puncture diameters apart. Color. Body black, without metallic highlights; coxae and tarsi brown, femora and tibia basally brown, apically white; scape, pedicel brown to dark brown, flagellomeres I-III at least partly white, remainder of flagellomeres dark brown; wing membrane light brown tinted, with dark brown veins.

Distribution. Papua New Guinea: Morobe Prov., Bulolo, 900 m, 13/ii/-13/ iii/1979, J. Sedlacek; Tekadu, 100m, i/1-17/2000, Sears \& Binatung brigade; 7-9/ ii/2010, T. Sears \& Binatung brigade, $7^{\circ} 38^{\prime}$ S, 146 $34^{\prime}$ E; Lakekamu Basin, Ivimka Research Sta., 120 m 707'44"S, 146 30'E, MT; 20/ii-2/iii/2000, T. Sears, MT iv/1-20/2000, T. A. Sears; xi/2-8/1999, Heydon, Schiff \& Sears; Wau; 1100 m, $15 /$ ix/1964, J. Sedlacek, MT; Baiyer River, 6-25/ii/1979, 1100m, J. Sedlacek; Madang Prov., Mt. Wilhelm, 200m, 16-25/v/2013; 700m, 543'55.596"S, $145^{\circ} 15^{\prime} 7.9194 " E ;$ 23-24/v/2013, 1200m, 543'15.1464"S, 14516'10.1994"E; Wanang, ix/1622/2012, 5¹3'39.6114"S, 1454'46.92"E; East Sepik Prov., Amboin Patrol Post, Karawar Lodge, ii/1983 A. C. Messer; 19 males and 3 females were examined (AEI, BME, BPBM). 

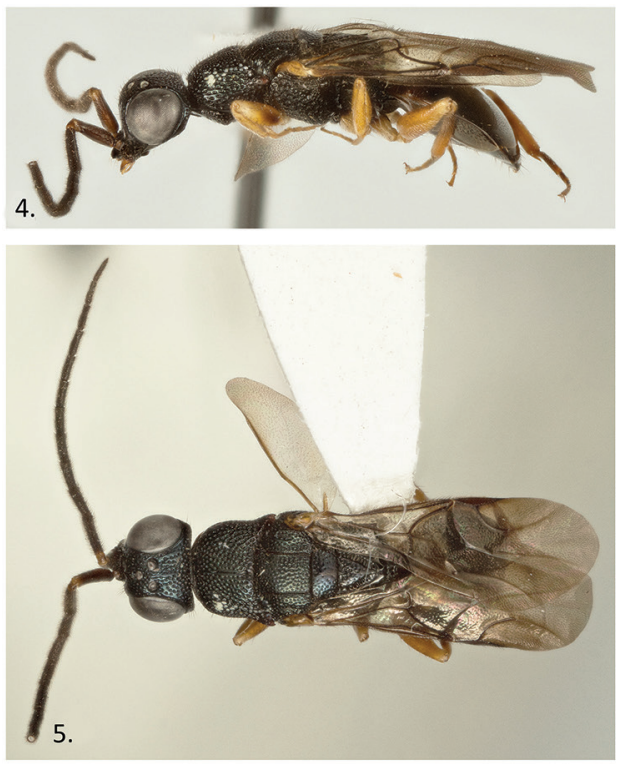

Cl. aquilus
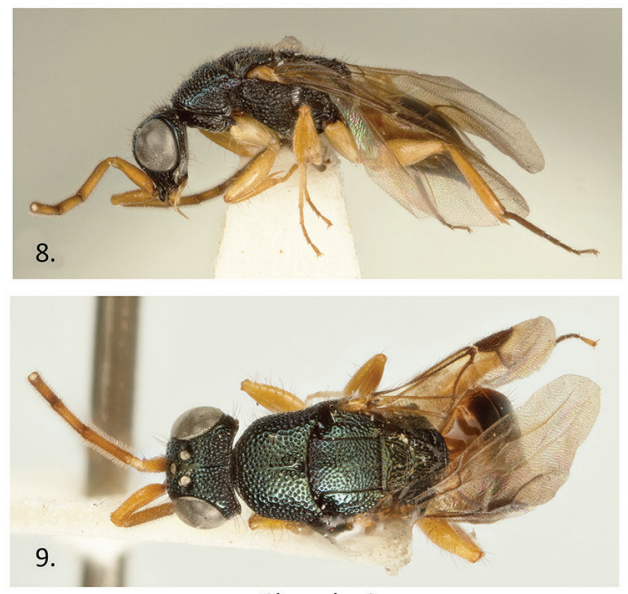

Cl. ceylonicus
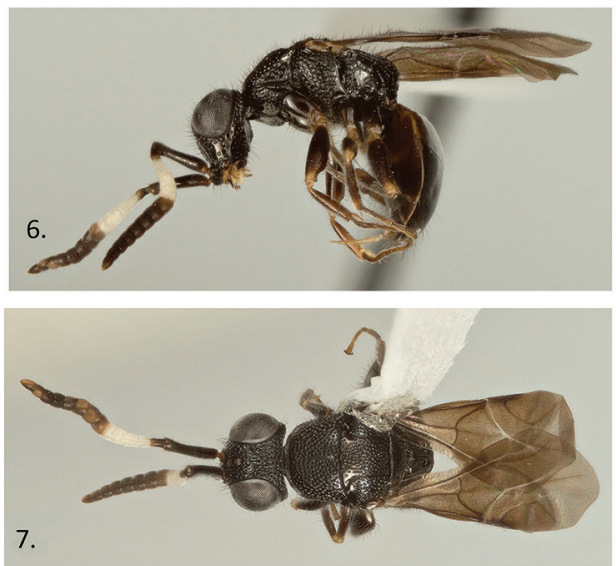

Cl. aquilus
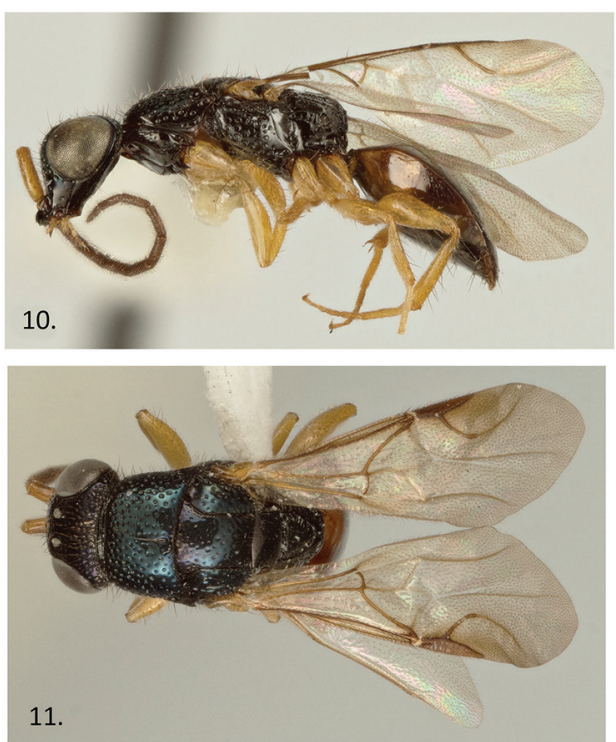

Cl. cruciger

Figures 4-I I. 4, 5, 8-I I Male Cladobethylus 6, 7 Female Cladobethylus 4, 6, 8, 10 Lateral view 5, 7, 9, I I dorsal view.

\section{Cladobethylus ceylonicus Krombein}

Figs 8, 9

Cladobethylus ceylonicus Krombein 1980: 253. Holotype male; Sri Lanka: Central Prov., Kandy Dist., Adams Peak (USNM).

Diagnosis. The most distinctive features of $\mathrm{Cl}$. ceylonicus are the long first and second flagellomeres, a characteristic shared with $\mathrm{Cl}$. acehensis and $\mathrm{Cl}$. densepunctatus. Howev- 
er, Cl. ceylonicus can be distinguished from these and other species by the combination of these features, along with the densely punctate second metasomal tergum, asymmetrical apical male flagellomeres and short subantennal distance.

Male description. Body (Figs 8, 9). Length $4.0 \mathrm{~mm}$. Head. Scapal basin impunctate, with short strip of cross-ridges on either side of narrow medial stripe; clypeus short and subtruncate apically, subantennal distance $0.4 \mathrm{MOD}$ long; malar space $3.8 \mathrm{MOD}$ long wide; head about as long as wide; interocular distance $1.9 \times$ eye width in front view; midocellus 2.2 MOD from ocular margin; ocelli arranged in isosceles triangle; hindocelli separated from ocular margin by 1 diameter; postocular distance 1.6 MOD wide; flagellomere I $4.5 \times$ as long as broad, setae $0.5 \times$ as long as flagellomere breadth; flagellomere II $3.3 \times$ as long as broad; flagellomere XI $5 \times$ as long as broad; flagellomeres VIII-X asymmetrically bulging submedially. Mesosoma. Pronotum about $1.1 \times$ as long as scutum; mesopleural punctures contiguous; metapleuron dorsally cross-ridged, ventrally polished, impunctate; propodeum laterally cross-ridged dorsally, ventrally polished, impunctate, with subrectangular posteromedial enclosure. Metasoma. Tergum I with few, widely scattered tiny punctures; tergum II with large patch of punctures 1-2 puncture diameters apart, separated by medial longitudinal stripe; terga III-V with dense, tiny, nearly contiguous punctures, with impunctate apical margin. Color. Body black, with metallic blue highlights; legs yellow; antenna orange; wing membrane brown-tinted, veins dark brown.

Female. Unknown.

Distribution. Sri Lanka: Rat Dist., Gilimale, Induruwa Jungle, Central Prov., Kandy Dist., Adams Peak. The holotype and one male paratype were examined.

\section{Cladobethylus cruciger Kieffer}

Figs 10, 11

Cladobethylus cruciger Kieffer 1922: 69. Syntype males; Philippines: Mindanao, Butan; Luzon, Laguna, Mt. Maquiling (Lost?).

Cladobethylus cruciger var. antennalis Kieffer 1922: 71. Holotype male; Philippines: Luzon, Tayabas, Mt. Banahao (Lost?). Synonymized by Kimsey and Bohart 1991. Cladobethylus coeruleus Kieffer 1922: 71. Holotype female; Philippines: Mindanao, Butan (Lost?), syn. nov.

Diagnosis. Cladobethylus cruciger males most closely resemble male $\mathrm{Cl}$. darlingi based on the cylindrical apical flagellomeres, nearly impunctate metasomal tergum II, and flagellomere I less than $3.5 \times$ as long as broad. It can be distinguished from $\mathrm{Cl}$. darlingi and other Cladobethylus species by the combination of the male least interocular distance equal to the eye width in front view, postocular distance one midocellar diameter wide, brown antenna and body with bluish highlights dorsally. There are insufficient characters given by Kieffer to distinguish female "Cl. coeruleus" from other female Cladobethylus.

Male description. Body (Figs 10, 11): length $4.0 \mathrm{~mm}$. Head. frons punctures 0.20.5 puncture diameters apart; scapal basin primarily smooth with short strip of cross- 
ridges on either side of broad medial stripe; clypeus long and truncate apically in front view, subantennal distance $1 \mathrm{MOD}$; malar space 3.6 MOD long; head $1.1 \times$ as long as wide in front view, interocular distance equal to eye width in front view; midocellus 2 MOD from ocular margin; ocelli arranged in nearly equilateral triangle; hindocelli separated from ocular margin by 1 diameter; postocular distance $1 \mathrm{MOD}$; flagellomere I 3.3-3.5x, setae half as long as flagellomere breadth; flagellomere II 2.7-3.0x; flagellomere IX 6x; apical flagellomeres cylindrical. Mesosoma. Pronotum about as long as scutum; mesopleural punctures $0.5-1.0$ puncture diameters apart; metapleuron smooth, impunctate; propodeum laterally smooth and impunctate, with posteromedial enclosure parallel-sided, subrectangular. Metasoma. Tergum I essentially impunctate; tergum II with few, tiny, widely scattered punctures; terga III-V with dense, contiguous to narrowly separated tiny punctures, with impunctate apical rim. Color. Body black, except dorsum of head, pronotum, scutum and scutellum with faint metallic blue highlights; legs including coxae yellow; antenna brown; wing membrane brown tinted; veins dark brown.

Female description (based on Kieffer's description of $\mathrm{Cl}$. coeruleus). Body length $4.0 \mathrm{~mm}$. Head. Flagellomere I 2.5x as long as broad; flagellomere II $1.5 \times$ as long as broad; flagellomere X more than twice as long as broad. Metasoma. Third tergum closely and rather finely punctured. Color. Body black, except mesosomal dorsum with metallic blue highlights; legs including coxae yellow.

Distribution. Philippines: Mindanao: Misamis Or., Dinawehan Gingoong, 26 km e Gingoong City, 100-300m, 12/viii/1965, L. Torrevillas; Butan; Luzon, Laguna, Mt. Maquiling; one non-type male has been seen (BPBM).

Discussion. The description above is based on Kieffer's descriptions of $\mathrm{Cl}$. coeruleus and $\mathrm{Cl}$. cruciger, plus a single male specimen from the island of Mindanao that fits Kieffer's description.

Kieffer's Cladobethylus coeruleus is synonymized here with $\mathrm{Cl}$. cruciger as his description of coeruleus matches the shared characteristics between male and female Cladobethylus seen in other species where both sexes are known. Both specimens are from Mindanao. In the female description, Kieffer refers to punctation of the third tergum, but it is not clear whether he counted the propodeum as the first tergum.

The subspecies $\mathrm{Cl}$. antennalis might be a valid species based on the differences in flagellomere dimensions and collection location on a separate island. In Kieffer's description flagellomere I is $3.5 \times$ along as broad and flagellomere II $3 \times$ as long as broad in Cl. cruciger s. s., but in Cl. antennalis flagellomere I is $3 \times$ as long as broad and II twice as long as broad. However, without access to the types it is impossible to be certain.

\section{Cladobethylus darlingi Kimsey, sp. nov.}

http://zoobank.org/71BED8B5-7E81-4C25-AA13-4E850E01A370

Figs 12-15

Diagnosis. Males of this species most closely resemble those of $\mathrm{Cl}$. cruciger as discussed under that species. It can be distinguished from $\mathrm{Cl}$. cruciger and other male 
Cladobethylus by the combination of the malar space 3.5 midocellar diameters wide or wider, interocular distance more than twice eye width in front view, flagellomere I less than $3.5 \times$ as long as broad, flagellomere II less than $2.5 \times$ as long as broad, and flagellomere XI more than $6.5 \times$ as long as broad.

Male description. Body (Figs 12, 13). Length 3.0-5.0 mm. Head. Frons with punctures 1-2 puncture diameters apart; scapal basin primarily smooth with narrow strip of cross-ridges on either side of broad medial stripe and scattered punctures laterally along eye margins; clypeus long, projecting ventrally, projection apicomedially irregularly truncate; subantennal distance 1.2 MOD long; malar space 3.8 midocellar diameters wide; head about as long as wide; interocular distance $2.2 \times$ eye width in front view; midocellus 2.4 diameters from ocular margin; ocelli arranged in nearly equilateral triangle; hindocelli separated from ocular margin by 1.0-1.2 diameters; flagellomere I 3.5-3.8x as long as broad, setae length $0.3 \times$ flagellar width; flagellomere II $2.3-2.5 \times$ as long as broad; flagellomere XI $6.7 \times$ as long as broad; apical flagellomeres asymmetrically slightly bulging medially. Mesosoma. Pronotum slightly shorter than scutum; mesopleural punctures 0.5 puncture diameters apart; metapleuron polished with 1-3 irregular transverse ridges; propodeum laterally polished, with subrectangular posteromedial enclosure. Metasoma. Tergum I with medial transverse zone of widely separated tiny punctures, otherwise impunctate; tergum II highly polished, with tiny punctures 2-10 puncture diameters apart, with impunctate longitudinal medial band and posterior margin; terga III-IV with tiny punctures, 1-3 puncture diameters apart, posterior margins impunctate. Color. Body black, except head vertex with purplish highlights and dorsum of pronotum, scutum, scutellum and metanotal triangle with metallic blue highlights; metasomal tergum I reddish basally, apically black, often with bluish highlights, terga II-V black, often with bluish highlights; legs including coxae yellow; antenna orange to light brown; wing membrane light brown-tinted, with dark brown veins.

Female description. Body (Figs 14, 15). Length $3.0 \mathrm{~mm}$. Head. Frons punctures contiguous to 0.5 puncture diameter apart; scapal basin impunctate, with narrow band of cross-ridges submedially; clypeus short, truncate apicomedially; subantennal distance $0.4 \mathrm{MOD}$ wide; malar space $4 \mathrm{MOD}$ long; head $0.9 \times$ as long as broad, interocular distance $1.1 \times$ eye width in front view; postocular distance $0.4 \mathrm{MOD}$; vertex rounded angulate posterolaterally in dorsal view; midocellus 2.2 MOD from ocular margin; ocelli arranged in isosceles triangle; hindocelli separated from ocular margin by 1 hindocellar diameter; flagellomere I $2.3 \times$ as long as broad; flagellomere II as long as broad; flagellomere X $1.8 \times$ as long as broad. Mesosoma. Mesopleural punctation contiguous; metapleuron polished with metapleural-propodeal suture foveate; propodeum smooth, impunctate below wing. Metasoma. Tergum I and II with tiny widely separated punctures, 10 puncture diameters apart or more; terga III and IV punctures 1-3 puncture diameters apart, with impunctate apical rim. Color. Body black, without metallic highlights; legs brown; antenna brown; wing membrane light brown tinted, with dark brown veins.

Type material. Holotype male: Indonesia: Borneo, West Kalimantan, Gunung Palung Nat. Park, Cabang Panti Res. Sta., 100m, Malaise trap, rainforest alluvial, $1^{\circ} 15^{\prime}$ S, $110^{\circ} 5^{\prime} \mathrm{E}, 15$ Jun-15 Aug, 1991, Darling, Rosichon, Sutrisno, IIS910122 

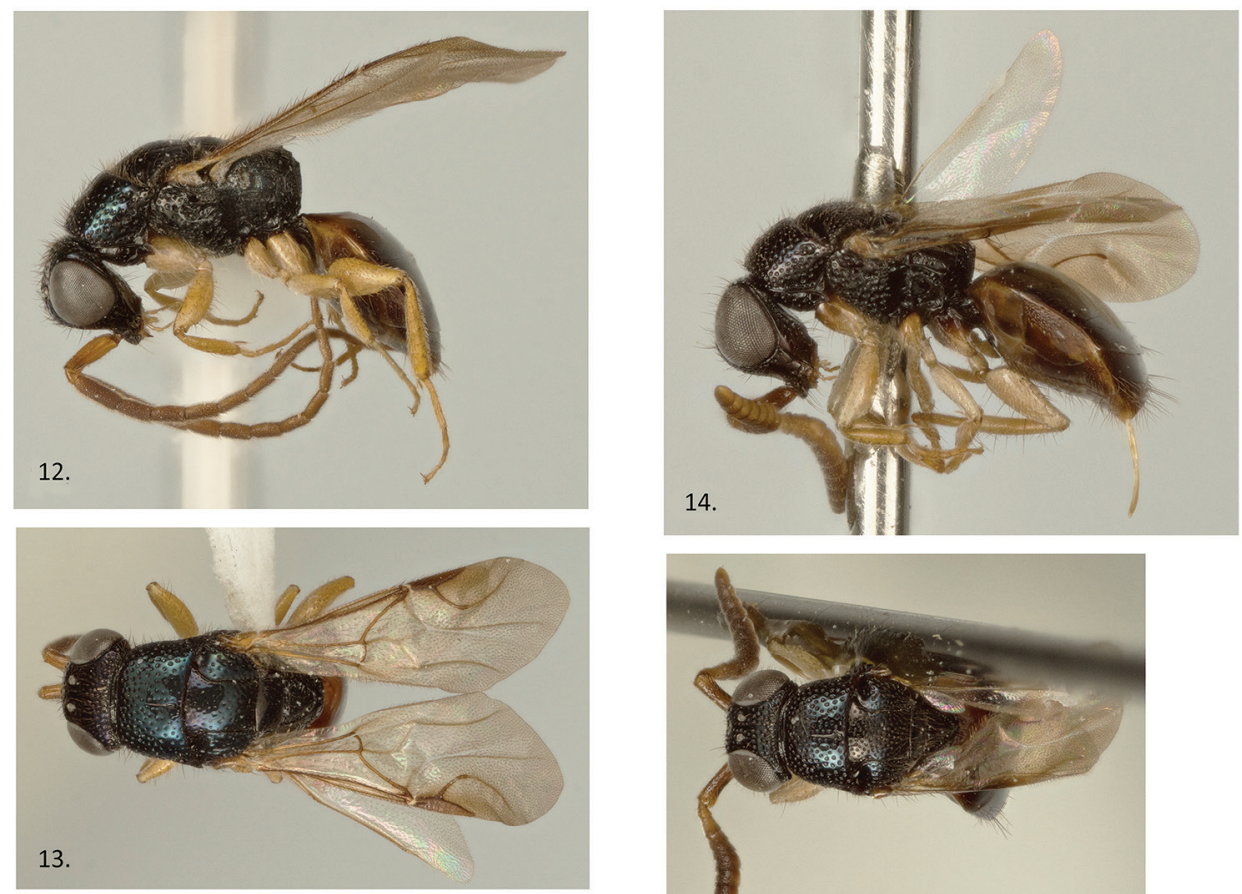

Cl. darlingi
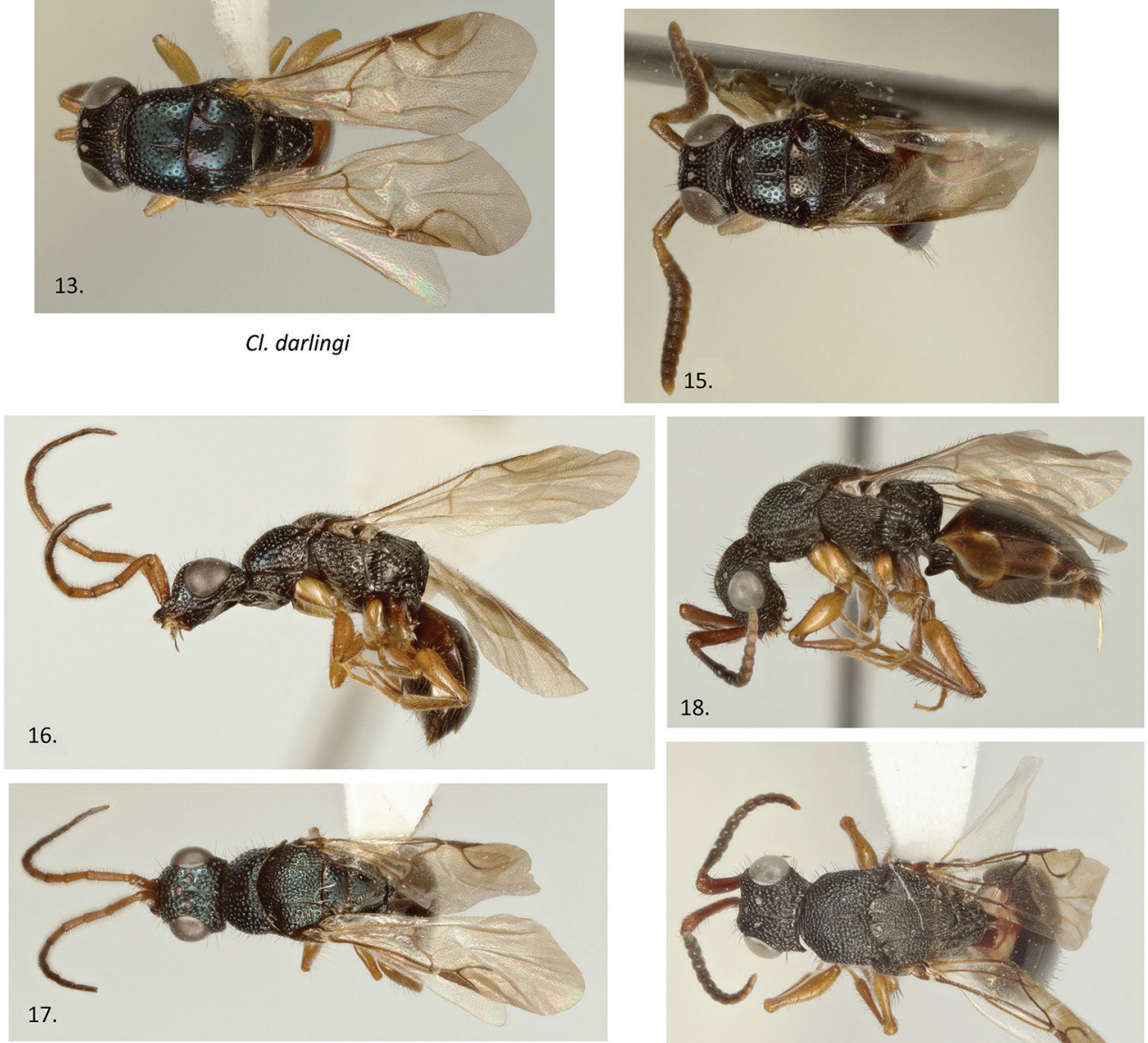

Cl. densepunctatus

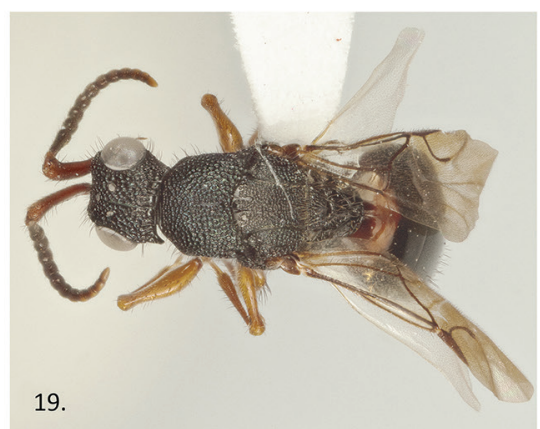

Figures 12-19. 12, 13, 16, 17 Male Cladobethylus 14, 15, 18, 19 Female Cladobethylus 12, 14, 16, 18 Lateral view 13, 15, 17, 19 dorsal view. 
(ROM). Paratypes: 8 males, 2 females; 3 males: same data as holotype; 2 males: same data, except rainforest sandstone, IIS910119; 1 male: rainforest sandstone, light gap, IIS910116; 1 male: 100-400m, rainforest sandstone, IIS910129; 1 male: 29/vi/1991, rainforest alluvial, screen sweep, IIS910165; 1 female: rainforest sandstone, Malaise trap pans, IIS9310134; 1 male: rainforest peat swamp, Malaise trap pans, IIS910132; 1 female: rainforest granite, Malaise trap pans, IIS910115; 1 male: 17-29/vi/1991, rainforest sandstone, Malaise trap, IIS910135 (BME, MZB, ROM).

Distribution. Borneo. Additional non-type material from Borneo includes three males: East Kalimantan, Kac. Pujungan, Kayan-Mentarang Nat. Res., 21/iii/-4/ iii/1992, $2^{\circ} 52^{\prime} \mathrm{N}, 115^{\circ} 49^{\prime} \mathrm{E}$, lowland Dipt. Forest, Lalut Birai riparian vegetation $378 \mathrm{~m}$, screen sweep, DC Darling, IIS930008; 4 males: Brunei, Kuala Belalong, FSC, 4³4'N, $115^{\circ} 7^{\prime} \mathrm{E}, 18 / \mathrm{v} / 1991$, Malaise, N. Mawdsley, BMNH(E)1991-173; 1 male: iii/1991, BMNH(E)1991-173; 1 male: Brunei, Ulu Temburong base camp, 300m, 16/ii-9/ iii/1982, M.C. Day; 1 male: North Borneo (SE), 19 km n, forest camp, 60 m, 21/ xi/1962, K.J. Kuncheria; 1 male: 7/xi/1862; 1 male: Sarawak, $4^{\text {th }}$ div., Gn. Mulu, RGS Exp. 17/xi/1923, D. Hollis, BM77-543; 1 male: British N. Borneo, Gomantong Caves, 22-26/xi/1958, TC Maa; 2 males: Borneo, Sarawak, sw Gunung Buda, 64km s Limbang, $4^{\circ} 13^{\prime} \mathrm{N}, 114^{\circ} 56^{\prime} \mathrm{E}, 22-28 / x i / 1996, \mathrm{MT}$, SL Heydon \& S Fung; 1 male: 8-15/xi/1996.

Etymology. The species is named after Chris Darling who not only collected this species, but also has collected many other Cladobethylus species in Southeast Asia.

\section{Cladobethylus densepunctatus Kimsey, sp. nov.}

http://zoobank.org/B76A5F6E-B578-401C-B63B-02F5171E7070

Figs 16-19

Diagnosis. This is the most distinctive of the Cladobethylus species. The coarse punctation and long, strongly angulate vertex will immediately separate $\mathrm{Cl}$. densepunctatus from all other species in both sexes. In addition, males have the apical flagellomeres asymmetrical, and females have the longest first flagellomere $(2.5 \times)$, widest interocular distance (more than twice eye width), longest subantennal distance (more than 1.3 midocellar diameters), lack occipital fovea (shared with $\mathrm{Cl}$. thailandicus) and have an entirely brown antenna.

Male description. Body (Figs 16, 17). Length 2.0-4.0 mm. Head. Scapal basin coarsely punctate, at most with small number of wrinkles basomedially; clypeus long and gently convex apicomedially; subantennal distance 1.2 MOD; malar space 3.5 MOD long; head slightly shorter than broad, interocular distance $2.4 \times$ eye width in front view; postocular distance 2.3 midocellar diameters; vertex strongly angulate posterolaterally in dorsal view; midocellus $2 \mathrm{MOD}$ from ocular margin; ocelli arranged in isosceles triangle; hindocelli separated from ocular margin by 1.2 diameters; flagellomere I $3.5 \times$ as long as broad, setae $0.4 \times$ as long as flagellomere breadth; flagellomere II $2.6 \times$ as long as broad; flagellomere IX $5 \times$ as long as broad; apical five flagellomeres slightly lobate submedially. Mesosoma. Pronotum about as long as scutum; mesopleu- 
ron with punctures contiguous t 0.5 puncture diameters apart; metapleuron polished, impunctate; propodeum laterally polished, impunctate, with posteromedial enclosure subrectangular. Metasoma. Tergum I with small submedial patch of punctures, 1-2 puncture diameters apart, otherwise impunctate; tergum II basal half with punctures, 1-2 puncture diameters apart, separated by impunctate medial stripe, apical part impunctate; terga III-V with dense basal band of punctures separated by impunctate longitudinal band, posterior margin impunctate. Color. Body black, without metallic highlights; legs including coxae orange to red; antenna dark reddish brown; wing membrane light brown tinted, with dark brown veins.

Female description. Body (Figs 18, 19). Length. 3.0-6.0 mm. Head. Scapal basin coarsely punctate, with band of wrinkles basomedially; clypeus long and rounded apically, subantennal distance 1.8 MOD long; malar space 5.2 MOD long; head slightly broader than long; midocellus 3.6 MOD from ocular margin; ocelli arranged in isosceles triangle; hindocelli separated from ocular margin by 2 diameters; flagellomeres rounded, broadest submedially; flagellomere I 2.6x as long as broad; flagellomere II as long as broad; flagellomere X twice as long as broad. Mesosoma. Pronotum slightly shorter than scutum; mesopleural punctures large, contiguous; metapleuron with coarse cross ridging; propodeum laterally wrinkled, with posteromedial enclosure subrectangular. Metasoma. Tergum I impunctate, except for narrow transverse band of punctures separated by $0.5-1.0$ puncture diameters; tergum II with large ovoid basal punctate areas, with punctures $0.5-1.0$ puncture diameters apart, separated medially by impunctate longitudinal stripe, laterally and posteriorly nearly impunctate; terga III-V with punctures $0.5-1.0$ puncture diameters apart, with impunctate apical margin. Color. Body black, without metallic highlights; legs including coxae yellow; antenna: scape, pedicel red, flagellum dark brown becoming red ventrally toward apex; wing membrane light brown tinted, veins dark brown.

Type material. Holotype male: Thailand: Lampang Prov., Chae Son NP, Huai Yen, 18 50'012"N, 99 28'656"E, 419m, pan trap, 7-8/v/2008, Kwannui \& Sukpeng, T5304 (QSBG). Paratypes: 6 males, 9 females: 1 male: Bangkok, Khao Yai NP, 15/iv/1990, Black light, B.V. Brown; 1 female; Chiang Mai Prov., Doi Chang Dao NP, Pha Tang substation, 19²5'N, 98 55'E, 526 m, Songkran \& Apichart, 21-28.x.2007, MT, T3180; 1 female: Jugeau \& Watwanich, 8-15/iv/2008, T5791; 1 female: Doi Inthanon NP, Vachiratharn Falls, $18^{\circ} 32.311^{\prime} \mathrm{N}, 98^{\circ} 36.048^{\prime} \mathrm{E}, 700 \mathrm{~m}$, Malaise trap, 2-8.vii.2006, Y. Areeluck. T51; 1 female: Chiang Mai Botanic Garden, 18.8955N, 98.8636E, 11-25/vii/2013, M. Hauser; 1 female: Chaiyaphum Prov., Pa Hin Ngam NP, Thepana waterfall, $15^{\circ} 38.948^{\prime} \mathrm{N}, 101^{\circ} 25.625^{\prime} \mathrm{E}, 604 \mathrm{~m}$, Malaise trap, 7-13.x.2006, Sa-nog \& Adnafai, T660; 1 male: Chanthaburi Prov., Khao Khitchatkut NP, $12^{\circ} 50.2^{\prime} \mathrm{N}, 102^{\circ} 7.3^{\prime} \mathrm{E}, 46 \mathrm{~m}, 10-17 / \mathrm{IV} / 2009$, S. Charoenchai, MT, T4878; 1 female: Loei Prov., Phu Ruea NP, $17^{\circ} 27.901^{\prime} \mathrm{N}, 101^{\circ} 21.301^{\prime} \mathrm{E}, 700 \mathrm{~m}$, Malaise trap, 5-12.xii.2006, Patikom Tumtip, T1263; 1 male, 1 female: Phu Kradeung NP, 273m, $16^{\circ} 56.589^{\prime} \mathrm{N}, 101^{\circ} 42.074 \mathrm{E}$, 21/x/2006, S. Glonglasae, T786; 1 male: $16^{\circ} 49.9^{\prime} \mathrm{N}$, $101^{\circ} 47.6 ' E, 273 \mathrm{~m}$, Malaise trap, 14-21/v/2008, T. Phatai, T5011; 1 female: Sakon Nakhon Prov., Phu Phan NP, 1648.628'N, 10353.591'E, 522m, Malaise trap, 3-9. 
xii.2006, S. Kongnara, T1241; 1 male: Surat Thani, Khao Sok NP, Ban Huaraed, $8^{\circ} 54.555^{\prime} \mathrm{N}, 98^{\circ} 30.522^{\prime} \mathrm{E}, 122 \mathrm{~m}, \mathrm{MT}, 6-13 / \mathrm{I} / 2009$, Pongphan, T3913; 1 male: Trang Prov., Khaophappha Khaochang, 200-400m, 10.1.1964, W. Samuelson; 1 female: Ubon Ratchathani Prov., Pha Taem NP, $15^{\circ} 37.321^{\prime} \mathrm{N}, 105^{\circ} 36.982^{\prime} \mathrm{E}, 419 \mathrm{~m}$, Malaise trap, 13-20.x.2006, T723 (BME, CHIANG MIA).

Distribution. Thailand: Bangkok, Chiang Mai, Chaiyaphum Chanthaburi, Loei, Sakon Nakhon, Surat Thani, Trang, Ubon Ratchathani Provinces. One additional male was seen from Viet Nam: 6 km s Dalat, 1400-1500 m, 9/vi-7/vii/1961, N. R. Spencer.

Etymology. The species is named for the unusually dense, coarse punctation on the head and thorax.

\section{Cladobethylus gilbus Kimsey}

Figs 20-25

Cladobethylus gilbus Kimsey 1986:157. Holotype male; Malaysia: Pasoh Forest Res., Negri Sembilan (AEI).

Diagnosis. Cladobethylus gilbus shares the nearly impunctate second metasomal tergum with $\mathrm{Cl}$. darlingi, $\mathrm{Cl}$. acehensis and $\mathrm{Cl}$. densepunctatus. In males it can be distinguished from those species by the narrow malar space (less than 2.8 midocellar diameters), flagellomere I less than $4 \times$ as long as broad, flagellomere II more than $2.5 \times$ as long as broad, flagellomere XI $7 \times$ as long as broad and postocular distance less than 1 MOD. Females have the basal three flagellar segments paler than the rest of the flagellum, very short flagellomeres I and II and malar space 4.5 midocellar diameters wide (shared with $\mathrm{Cl}$. aquilus).

Male description. Body (Figs 21, 22). Length 3.5-4.0 mm. Head. Scapal basin with narrow band of cross-ridges separated by smooth medial band; head venter without ovoid foveae along midline of gular area, gular area punctate; malar space 2.6 MOD long; head as wide as long; interocular distance $1.5 \times$ eye width in front view; midocellus 1.4 MOD from ocular margin; ocelli arranged in a nearly equilateral triangle; hindocelli separated from ocular margin by 1 diameter; subantennal distance 0.6 MOD; clypeal apex truncate to slightly concave; flagellomere I $3.6 \times$ as long as broad, setae length $0.4 \times$ flagellomere breadth; flagellomere II $2.6 \times$ as long as broad; flagellomere IX $7 \times$ as long as broad; flagellomeres VIII-X slightly lobate submedially. Mesosoma. Pronotum $0.9 \times$ as long as scutum; mesopleural punctures $0.2-0.5$ puncture diameters apart; metapleuron with zone of cross ridging below hindwing base; propodeum laterally cross-ridged, posteromedially with subrectangular enclosure. Metasoma: terga I and II impunctate; terga III-V with basal band of dense, minute, contiguous punctures. Color: body black, except pronotum, scutum, scutellum and metanotal triangle with metallic blue highlights; antenna and legs including coxae yellow; wing membrane light brown tinted, with brown veins.

Female description. Body (Figs 23, 24). Length. 4.0-5.0 mm. Head. Frons punctures contiguous to 0.5 puncture diameters apart; scapal basin primarily smooth with 


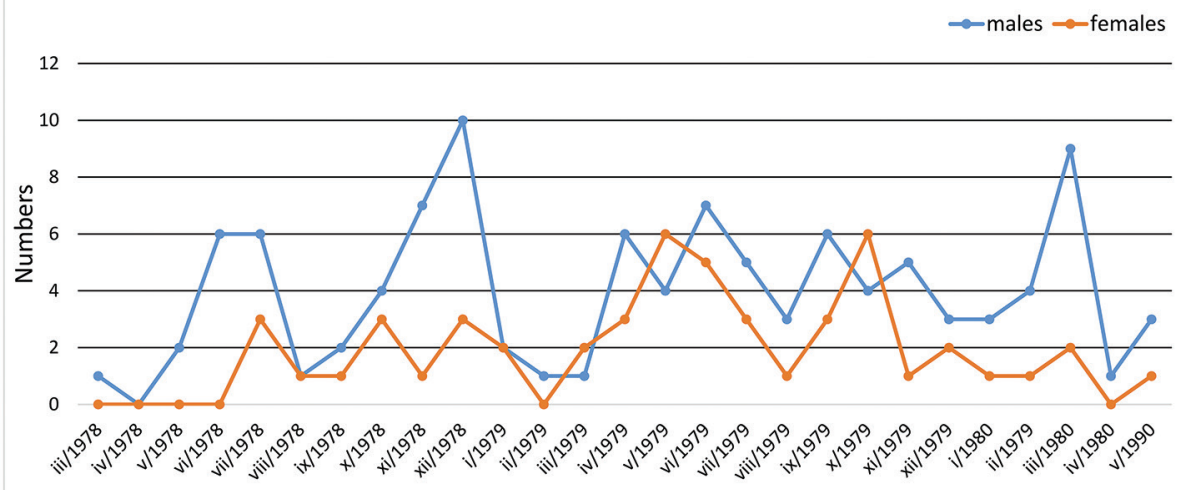

20.
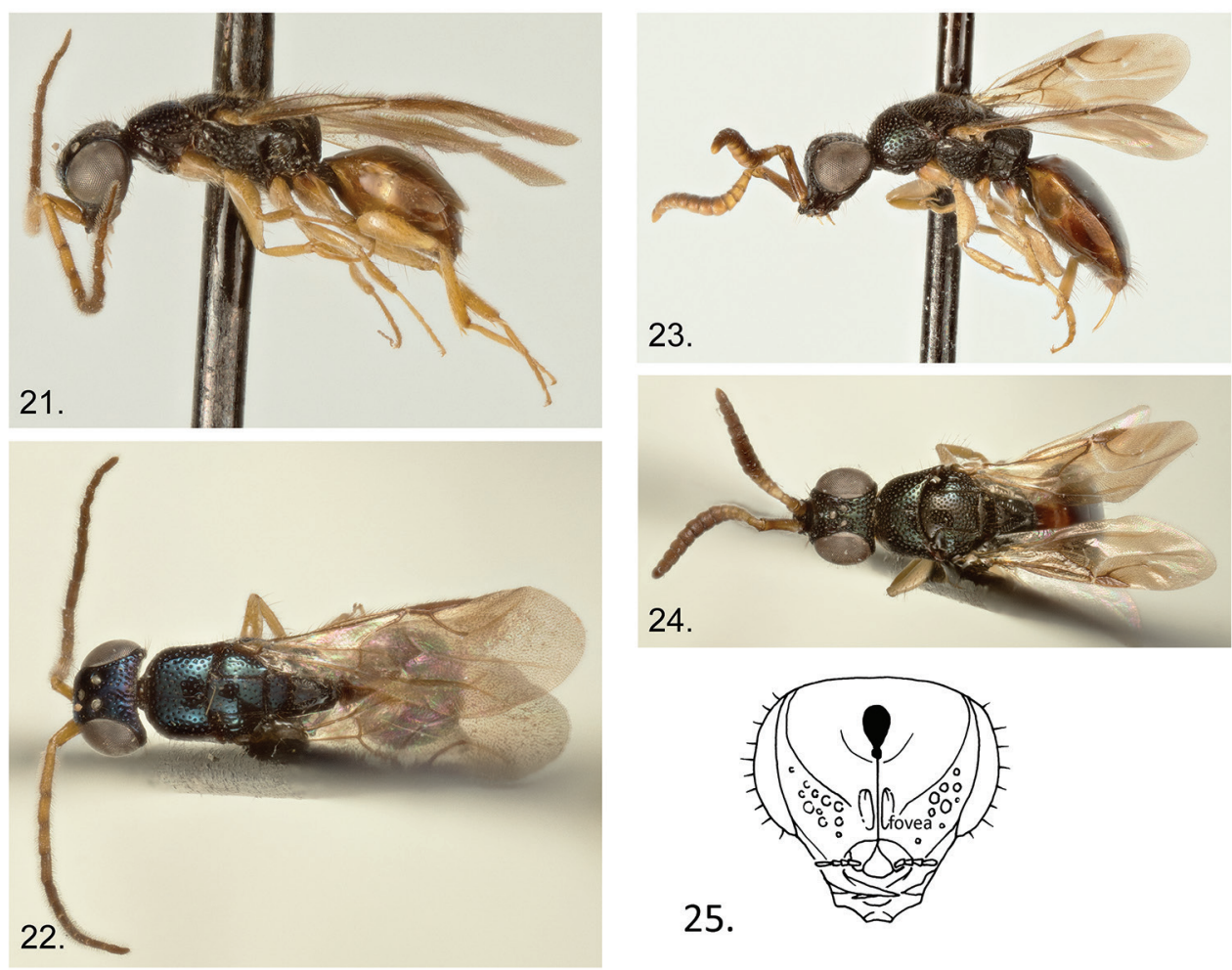

Figures 20-25. Cladobethylus gilbus 20 graph of seasonal abundance of males and females in Malaysia 21, 22 males 23-25 females 21, 23 lateral view 22, $\mathbf{2 4}$ dorsal view $\mathbf{2 5}$ ventral view of head.

short strip of cross-ridges on either side of broad medial stripe; head venter with ovoid fovea on either side of gular midline (Fig. 25), gular area punctate; clypeus subtruncate apically; subantennal distance 0.8 midocellar diameter; malar space 4.5 midocellar diameters long; head about as long as wide; midocellus $2 \times$ midocellar diameters from ocu- 
lar margin; ocelli arranged in nearly equilateral triangle; hindocelli separated from ocular margin by one diameter; flagellomere I as long as broad; flagellomere II $0.6 \times$ as long as broad; flagellomere $\mathrm{X}$ twice as long as broad. Mesosoma. Pronotum about as long as scutum; mesopleuron with irregular scrobal sulcus, punctures contiguous to 0.5 puncture diameter apart; metapleuron smooth and impunctate; propodeum laterally polished, impunctate, posteromedially with subrectangular enclosure smooth but somewhat irregular. Metasoma. Tergum I impunctate; tergum II punctures 10 puncture diameters apart becoming slightly denser laterally; terga II-IV punctures 1-2 puncture diameters apart with impunctate rim. Color. Body black, except head and pronotum with faint metallic blue highlights; legs including coxae yellow; scape yellowish brown becoming darker ventrally; pedicel and flagellomeres I-III yellow, remaining flagellomeres brown dorsally becoming yellow ventrally; wing membrane brown-tinted, veins dark brown.

Distribution. MALAYSIA: Negeri Sembilan, Pasoh Forest Res.; 106 males and 50 females were examined (AEI, BME). Males and females were found in nearly every month over a two-year period of Malaise trap collecting at this site (Fig. 20).

\section{Cladobethylus insularis Kimsey \& Dewhurst}

Figs 26-29

Cladobethylus insularis Kimsey, Dewhurst \& Nyaure, 2012: 3. Holotype male; Papua New Guinea, Northern Prov., Higaturu (BMNH).

Diagnosis. This is the largest bodied species of Cladobethylus, with males averaging about $1.5 \times$ the length of the known other species. Male $\mathrm{Cl}$. insularis can be distinguished from $\mathrm{Cl}$. aquilus, the only other species known from New Guinea by the pronotum without blue tints, a much broader zone of cross-ridging in the scapal basin, legs brown instead of yellow ( $\mathrm{Cl}$. aquilus) flagellomere I shorter ( $4 \times$ as long as broad versus $5 x$ in $\mathrm{Cl}$. aquilus), and flagellomere XI shorter $(5 x$ as long as broad versus $6 \times$ in $\mathrm{Cl}$. aquilus). Features of female $\mathrm{Cl}$. insularis not shared with other Cladobethylus include the bicolored antenna and legs, long clypeus (1 MOD long versus 0.3-0.6 $\mathrm{MOD}$ in other species), and narrow distance between the midocellus and nearest eye margin (up to $2 \mathrm{MOD}$ versus 2.6-2.7 MOD in the others).

Male description. Body (Figs 26, 27). Length 5.0-7.0 mm. Head. Face about as long as broad across the eyes; genal area without foveae; midocellus 1.8 diameters from ocular margin; hindocellus 0.9 diameters from ocular margin; scapal basin with narrow, longitudinal submedial band of cross-ridges; malar space 3.5-3.8 MOD; subantennal distance $1 \mathrm{MOD}$ long; interocular distance twice as wide as eye width in front view; postocular distance from ocular margin $1.8 \mathrm{MOD}$ wide, postocular margin sharply angulate posterolaterally; flagellomere I $4.0-4.2 \times$ as long as broad; flagellomere II 2.6-3.0 $\times$ as long as broad, setae length $0.5 \times$ as flagellomere breadth; flagellomere IX 5.5-5.7× as long as broad; ocular setulae 0.4 MOD long. Mesosoma (Fig 9). Pronotum about as long as scutum; punctation on pronotum, scutum and mesopleuron large, 

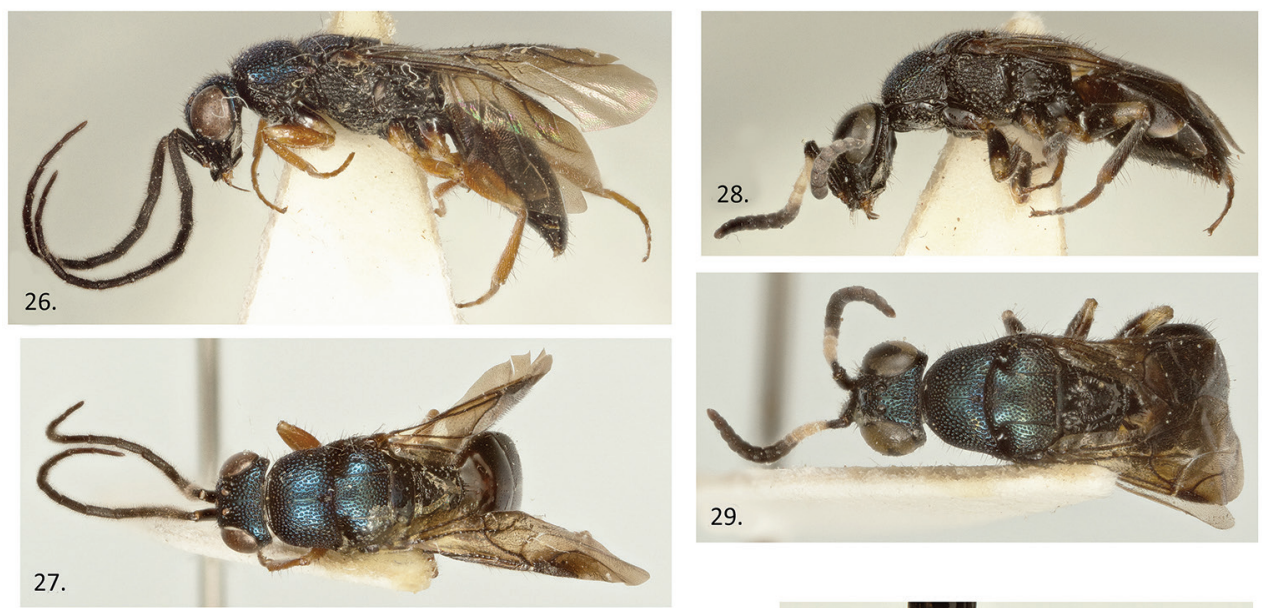

\section{Cl. insularis}
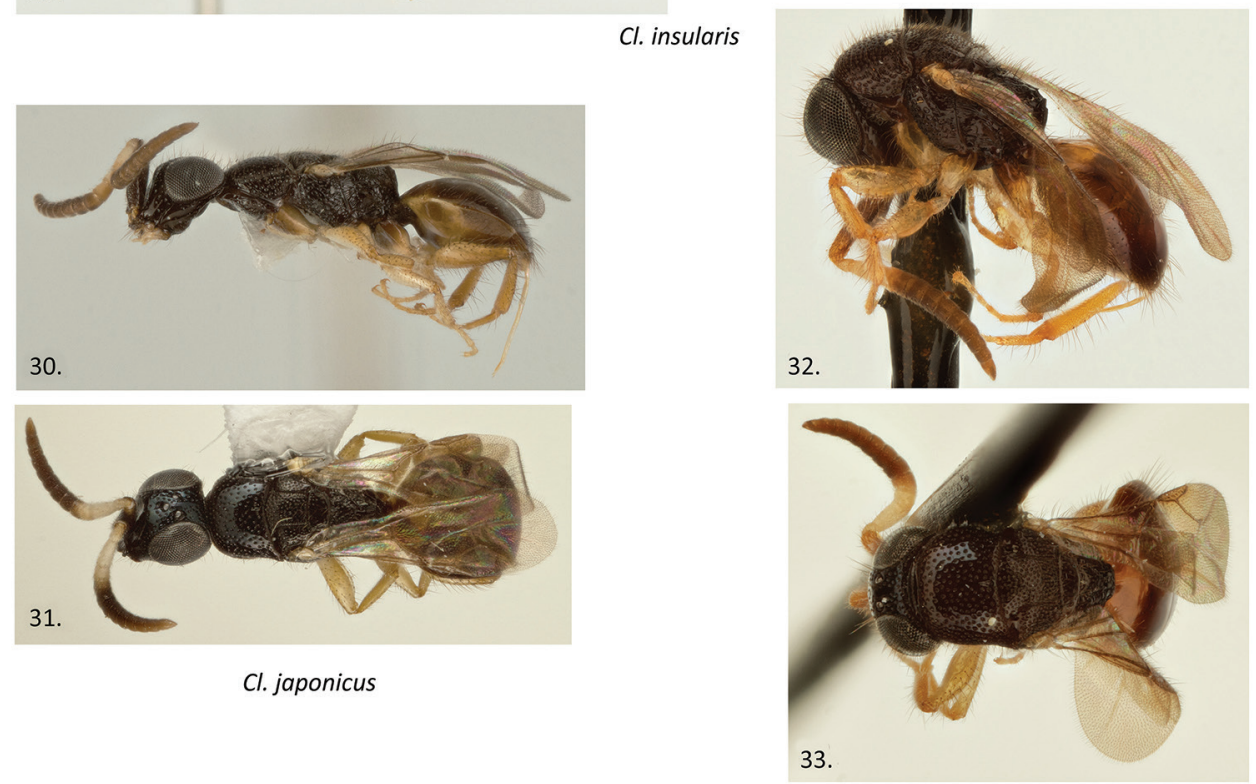

Cl. koreensis

Figures 26-33. 26, 27 Male Cladobethylus 28-33 female Cladobethylus 26, 28, 30, 32 lateral view 27, 29, 3 I, 33 dorsal view.

deep and nearly contiguous; with scutellum polished with scattered tiny punctures; metapleuron polished, impunctate; propodeum laterally polished and impunctate with several cross ridges ventrally, posteromedial enclosure broadly rectangular, polished and impunctate medially. Metasoma. Tergum I with transverse medial band of tiny punctures $2-4$ puncture diameters apart, impunctate otherwise; tergum II with two large ovoid patches of small punctures, contiguous to 1 puncture diameter apart, separated medially by impunctate band, with broad apical impunctate band; terga III-IV with tiny punctures 1 puncture diameter apart, becoming sparser toward apical margin. Color. Body black, with metallic bluish green highlights dorsally on head, pro- 
notum and scutellum, sometimes faintly on metasomal terga; mandible and antenna dark brown; legs light reddish brown, except coxae dark brown to black basally; wing membrane brown-tinted, darkest in vicinity of stigma; wing veins dark brown.

Female description. Body (Figs 28, 29). Length. 4.5-5.5 mm. Head. Malar space 3.6-3.8 MOD wide; subantennal distance 0.6 MOD wide; interocular distance $1.2 \times$ as wide as eye in front view; postocular distance 1.1 MOD wide, posterolateral margin broadly angulate; midocellus 2 diameters from ocular margin; hindocellus 1 diameter from ocular margin; flagellum fusiform, flagellomeres broadest apically, flagellomere I 2.2-2.4x as long as broad, flagellomere II as long as broad, flagellomere X 1.3-1.5x as long as broad. Mesosoma. Pronotum $1.2 \times$ as long as scutum medially; mesopleural punctures contiguous; metapleuron smooth, impunctate; propodeum laterally smooth, impunctate, posteromedial enclosure subrectangular. Metasoma: tergum I with transverse medial band of scattered tiny punctures, otherwise impunctate; tergum II punctures $0.5-1.0$ puncture diameters apart, with impunctate medial longitudinal band and posterior margin; tergum III-V punctures tiny, 0.5-1.0 puncture diameter apart with impunctate margin. Color: scape, pedicel black; flagellomeres I-III white, remaining flagellomeres black; coxae, femora, mid and hindtibiae black basally, whitish apically; foretibia and tarsi brown; wing membrane brown-tinted with dark brown veins.

Distribution. Papua New Guinea, Northern (Oro) Province, Higaturu; 18 males, 6 females; ex Eurycantha insularis eggs (BME, BMNH).

\section{Cladobethylus japonicus Kimsey}

Figs 30, 31

Cladobethylus japonicus Kimsey 1986: 158. Holotype female; Japan: Kyoto (AEI).

Diagnosis. This species has several unusual diagnostic female features. The eye has very long dense setulae, the hindocelli are very close to the ocular margins and the malar space is very long. In addition, the pronotum is longer than the scutum, and the metapleuron is cross-ridged from wing base to coxa.

Male. Unknown.

Female description. Body (Figs 30, 31). Length: $2.5 \mathrm{~mm}$. Head. Scapal basin smooth with short strip of cross-ridges on either side of broad medial stripe; clypeus short, broadly rounded; subantennal distance 0.6 MOD; malar space $5 \mathrm{MOD}$ long; interocular distance 1.5-1.7× eye width in front view; face about as broad as long in front view; ocular setulae $1 \mathrm{MOD}$ long or longer; midocellus 2.6 MOD from ocular margin; hindocellus separated from ocular margin by one diameter; ocelli arranged in nearly equilateral triangle; hindocelli separated from ocular margin by 1 hindocellar diameter; flagellomere I 2.3× as long as broad; flagellomere II $1.4 \times$ as long as broad. Mesosoma. Mesopleural punctures larger than on pronotum; metapleuron cross-ridged from hindwing base nearly to midcoxa; propodeum with posteromedial enclosure rough, subrectangular. Metasoma: terga I-V with tiny scattered punctures at least 4-6 PD apart laterally, nearly impunctate medially. Color: body black with bluish tints on vertex and 
pronotum; legs including coxae yellow; scape dark brown becoming paler distally; pedicel and flagellomeres I-III yellow, remaining flagellomeres brown; mandibles brown.

Distribution. JAPAN: Kyoto; 1 female (the holotype) examined (AEI).

\section{Cladobethylus koreensis Kimsey, sp. nov.}

http://zoobank.org/3B10AB85-C52F-4AD8-A2F7-DD6EC718CFE5

Figs 32, 33

Diagnosis. This species has several unusual female features. The eye has very long dense setulae, the hindocelli are very close to the ocular margins and the malar space is very long. In addition, the pronotum is longer than the scutum, and the metapleuron is cross-ridged from wing base to coxa.

Male. Unknown.

Female description. Body (Figs 32, 33). Length. 2.5-3.0 mm. Head. Scapal basin smooth, polished with narrow strip of cross-ridges on either side of broad medial stripe; clypeus short, broadly convex apicomedially; subantennal distance $0.5 \mathrm{MOD}$ wide; malar space $5 \mathrm{MOD}$ long; interocular distance $1.2 \times$ eye width in front view; face about $3 \times$ as long as broad across midocellus in front view; ocular setulae 1.5 MOD long or longer; midocellus $3 \mathrm{MOD}$ from ocular margin; hindocellus separated from ocular margin by 1 hindocellar diameter; ocelli arranged in isosceles triangle; postocular distance $0.5 \mathrm{MOD}$; flagellomere I $2.3 \times$ as long as broad; flagellomere II as long as broad; flagellomere X $1.5 \times$ as long as broad. Mesosoma. Mesopleural punctures larger than on pronotum, $0.2-1.0$ puncture diameters apart; metapleuron impunctate medially, transversely ridged, with smooth area medially; propodeum laterally impunctate and irregularly wrinkled, posteromedial enclosure ovoid. Metasoma: terga I-V with tiny scattered punctures at least $10 \mathrm{PD}$ apart laterally, nearly impunctate medially. Color: body black without bluish tints; legs including coxae yellow; scape dark brown becoming paler distally; pedicel and flagellomeres I-III white, remaining flagellomeres brown; mandibles brown; wing base white, rest of wing membrane and veins brown.

Type material. Holotype female: South Korea: Jeollanam-Do, Wando Arboretum, 34.3585N, 126.6685E, 10/viii/2015, T-S. Kwan, forest Malaise trap (BME). Paratype female: same data as holotype.

Distribution. South Korea: South Jeolla Prov.

Etymology. The species is named for the country of collection.

\section{Cladobethylus thailandicus Kimsey, sp. nov.}

http://zoobank.org/6ECB75D0-B471-4061-BDBA-27E048EB3C68

Figs 34-37

Diagnosis. This is a distinctive species. Males can be distinguished by flagellomere XI $5 \times$ as long as broad (shared with $\mathrm{Cl}$. ceylonicus and $\mathrm{Cl}$. densepunctatus), scapal basin without cross-ridging, subantennal distance more than 1 midocellar diameter long and 
body lacking metallic bluish highlights. Females have a bicolored antenna, with the basal segments whitish and the apical segments dark brown, the postocular distance is less than one midocellar diameter, and the gular bridge lacks occipital foveae.

Male description. Body (Figs 34, 35). Length 2.5-4.0 mm. Head. Frons punctures 1-2 puncture diameters apart; scapal basin primarily smooth with short strip of cross-ridges on either side of broad medial stripe; clypeus long and truncate or slightly emarginate apically; subantennal distance 1.2 midocellar diameters; malar space 3.5 midocellar diameters long; head about as long as wide; midocellus 1.7 diameters from ocular margin; ocelli arranged in nearly equilateral triangle; hindocelli separated from ocular margin by one diameter; postocular distance 2.5 midocellar diameters; flagellomere I $4 \times$ as long as broad, setae $0.5 \times$ as long as flagellomere breadth; flagellomere II $3.2 \times$ as long as broad; flagellomere IX $5 \times$ as long as broad. Mesosoma. Pronotum about $0.9 \times$ as long as scutum; mesopleural punctures $0.5-1.0$ puncture diameters apart; metapleuron smooth, impunctate; propodeum laterally wrinkled, with posteromedial enclosure subrectangular. Metasoma. Tergum I essentially impunctate; tergum II with few, widely scattered, tiny punctures; terga III-V with dense, tiny punctures $0-0.5$ puncture diameters apart, with impunctate apical margin. Color. Body black, except pronotum, scutum, scutellum and medial triangle of metanotum with metallic blue highlights; legs including coxae yellow to orange; antenna brown; wing membrane brown, veins dark brown.

Female description. Body (Figs 36, 37). Length $2.5-4.0 \mathrm{~mm}$. Head. Frons punctures contiguous to 0.5 puncture diameters apart; scapal basin primarily smooth with short strip of cross-ridges on either side of broad medial stripe; clypeus long and apically truncate or slightly emarginate; subantennal distance 0.8 midocellar diameter long; malar space 3.8 midocellar diameters long; head as long as wide; midocellus 2 diameters from ocular margin; ocelli arranged in nearly equilateral triangle; hindocelli separated from ocular margin by $0.5-0.8$ diameters; postocular distance $0.5 \mathrm{MOD}$; flagellum fusiform; flagellomere I $2.4 \times$ as long as broad; flagellomere II $0.7-0.9 \times$ as long as broad; flagellomere X twice as long as broad. Mesosoma. Pronotum about as long as scutum; mesopleural punctures contiguous to 0.5 puncture diameter apart; metapleuron entirely cross-ridged; propodeum laterally wrinkled, with posteromedial enclosure subrectangular. Metasoma. Terga I and II largely impunctate with narrow lateral band of tiny punctures, 3-6 puncture diameters apart; tergum III with tiny punctures 1-2 puncture diameters apart, separated medially by impunctate longitudinal stripe and apical impunctate rim; tergum IV punctures tiny, separated by $0.5-1.0$ puncture diameters with narrow impunctate rim. Color. Body black, except pronotum and scutum with faint blue highlights; legs including coxae yellow; scape, pedicel and flagellomeres I-III pale orange to whitish, remaining flagellomeres brown, except tip of apical flagellomere whitish; wing membrane brown tinted, with dark brown veins.

Type material. Holotype male: Thailand: Nakon Nayok Prov., Khao Yai NP, Lam Takong view point, $14^{\circ} 25.762^{\prime} \mathrm{N}, 101^{\circ} 23.527^{\prime} \mathrm{E}, 732 \mathrm{~m}, 5-12 / \mathrm{x} / 2006$, W. Sook Kho, T741 (QSBG). Paratypes. 7 males, 10 females. 1 female: Nakhon Nayok Prov., Khao Yai, $14^{\circ} 24.619^{\prime} \mathrm{N}, 101^{\circ} 22.708^{\prime} \mathrm{E}, \mathrm{MT}, 19-26 / v i i / 2006$, P. Sandao, T148; 1 female: 

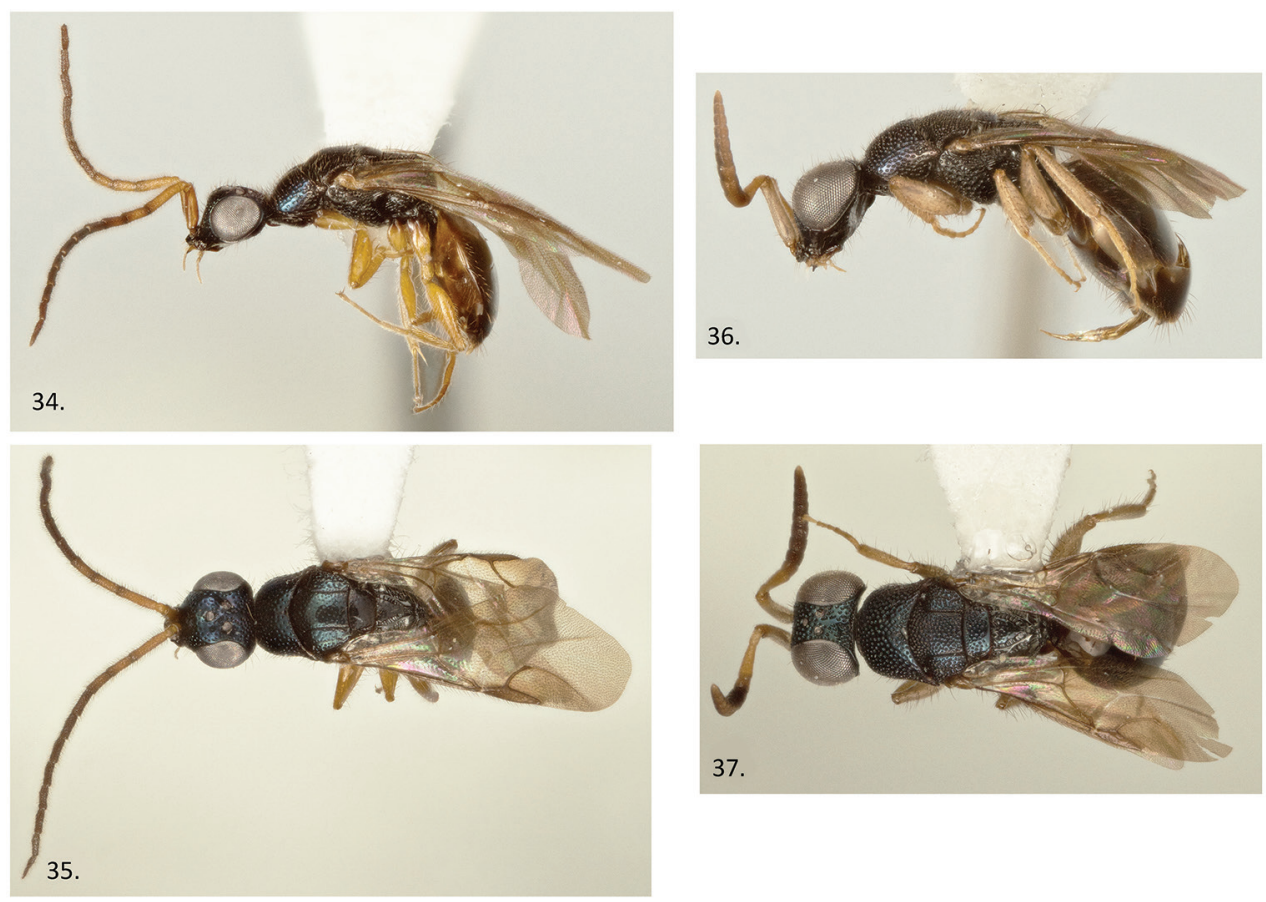

Figures 34-37. 34, 35 Male Cladobethylus thailandicus 36, 37 Female Cladobethylus 34, 36 lateral view 35,37 dorsal view.

Khao Yai NP, 6/vi/1965, P. D. Ashlock; 1 males, 2 females: 180 km NE Bangkok, Khao Yai NP, 780m, 11-18/iv/1990, MT, B.V. Brown; 2 males, 1 female: Nakhon Si Prov., Thammarat, Namtok Yong NP, 8¹4.3'N, 9948.3'E, 952m, Malaise trap, Paiboon, 30/ iii-6/iv/2009, T4658; 1 male: Thammarat, Namtok Yong NP, road to Khao Mhen 150m from Nern 499, $8^{\circ} 16.959^{\prime} \mathrm{N}, 99^{\circ} 39.149^{\prime} \mathrm{E}$, Malaise trap, 8-15/vi/2008, S. Samnaokan, T3095; 1 male: Trang Prov., Khaophappha Khaochang, 200m, 1-3/i/1964, GA Samuelson, Malaise trap, Bishop; 1 male: Chanthaburi Prov., Khao Khitchakut NP, Khao Prabaht Peak, $875 \mathrm{~m}, 12^{\circ} 50.4^{\prime} \mathrm{N}, 102^{\circ} 10^{\prime} \mathrm{E}$, MT, S. Charoenchai, 6-13/iii/2009, T4060; 1 male: Phetchabun Prov., Nam Nao NP, 16²43.695'N, 101³3.797'E, 921 m, YPT, 25-26/x/2006, N. Hongyothi, T1006; 1 female: Petchaburi Prov., Kaeng Krachan NP, 320m, 1247.9'N, 99²7.2'E, 27/vi-4/vii/2008, MT, Sirichai, T4338; 1 female: Nakhon Ratchasima Prov., Khao Yai NP, 700-800m, 18-24/iv/1990, MT, E. Fuller; 1 female; Tiger trail, $760 \mathrm{~m}, \mathrm{MT}, 14^{\circ} 27.5^{\prime} \mathrm{N}, 101^{\circ} 22^{\prime} \mathrm{E}, 26 / \mathrm{vi}-2 / \mathrm{vii} / 2007$, P. Sandao, T2232; 1 female: Chiang Mai Prov., Doi Chiangdao NP, Huai Na Lao, 500m, $19^{\circ} 24.731^{\prime} \mathrm{N}, 98^{\circ} 55.315^{\prime} \mathrm{E}, \mathrm{MT}, 3-9 / \mathrm{v} / 2008$, Jugsu \& Watwanich, T5803; P. Sandao, T2232; 1 female: Surat Thani Prov., Khao Sok NP, Bang Huaraed, 122m, $8^{\circ} 54.6^{\prime} \mathrm{N}$, 98³0.5'E, 5-12/v/2009, MT, Pongphan, T4861 (BME, QSBG).

Distribution. Thailand: Chanthaburi, Chiang Mai, Nakhon Nayok, Nakhon Si, Nakhon Ratchasima, Phetchabun, Phetchaburi, Surat Thani, and Trang Provinces.

Etymology. The species is named after the country of collection. 


\section{Acknowledgements}

This study was made possible by the individuals who collected these specimens as part of their studies, or had them in their collections and made them available to me for study, including Chris Darling, David Wahl, Brian Harris, Jim Boone and David Notton. In addition, particular thanks to Cheol Min Lee, from the Korea Forest Research Institute, Seoul, South Korea, for the Korean specimens.

\section{References}

Kieffer JJ (1922) Philippine Serphidae (Proctotrupidae). Philippine Journal of Science 20: 65-103.

Kimsey LS (1986) New species and genera of Asian Amiseginae. Psyche 93: 153-165. https:// doi.org/10.1155/1986/31631

Kimsey LS, Bohart RM (1991 [1990]) The Chrysidid Wasps of the World. Oxford Science Publications, New York, 652 pp.

Kimsey LS, Dewhurst CF, Nyaure S (2012) New species of egg parasites from the Oil Palm Stick Insect (Eurycantha insularis) in Papua New Guinea (Hymenoptera: Chrysididae; Phasmatodea: Phasmatidae). Journal of Hymenoptera Research 30: 19-28. https://doi. org/10.3897/jhr.30.4010

Krombein KV (1980) Biosystematic studies of Ceylonese wasps, I. A preliminary revision of the Amiseginae. PEP Deraniyagala Commemorative Volume (Sri Lanka): 246-260. 